\title{
Dieta e estratégia alimentar de Characidium rachovii (Characiformes, Crenuchidae) em riachos de planície costeira do sul do Brasil
}

\author{
Rodrigo F. Bastos ${ }^{1,2,3}$, Stéfanie F. Miranda ${ }^{1}$ \& Alexandre M. Garcia ${ }^{1,2}$ \\ 1. Laboratório de Ictiologia, Instituto de Oceanografia, Universidade Federal do Rio Grande (FURG). Av. Itália km 8, s/n ${ }^{\circ}$, Bairro Carreiros, 96203-900, Rio Grande, RS, Brasil. \\ (rfbastos@furg.br; amgarcia@mikrus.com.br) \\ 2. Programa de Pós-Graduação em Biologia de Ambientes Aquáticos Continentais, Universidade Federal do Rio Grande (FURG). \\ 3. Laboratório de Ecologia Aquática, Programa de Pós-Graduação em Biociências (Zoologia), Faculdade de Biociências (FABIO), Pontifícia Universidade Católica do Rio Grande do Sul \\ (PUCRS). Av. Ipiranga 6681, Prédio 12-C, sala 135-03, 90619-900, Porto Alegre, RS, Brasil.
}

\begin{abstract}
Diet and feeding strategy of Characidium rachovii (Characiformes, Crenuchidae) in coastal plain streams of southern Brazil. Characidium rachovii (Regan, 1913) is a frequent species in coastal plain streams on state of Rio Grande do Sul, southern Brazil. However, its trophic niche is still unknown. This paper aimed to: (i) describe the diet of C. rachovii in three coastal plain streams of RS; (ii) evaluate the influence of an ecotone (sea-stream) in the diet by comparing two sites, one near and other distant of the sea, and (iii) evaluate the effect of individuals number analyzed on the richness of the diet. The stomach contents analysis of 139 individuals revealed that the species was a specialist in Diptera (aquatic stage) and Amphipoda. Characidium rachovii was also opportunist, since besides such preference, consumed great richness of resources (24 items) and various with low frequency and high abundance. There were no differences in diet composition among streams and sites. The expected richness (rarefaction) showed that the number of individuals analyzed changes the estimate of diet richness in species that consume several items at low frequency.
\end{abstract}

KEYWORDS. Trophic niche, feed, rarefaction, coastal plain, coastal stream.

RESUMO. Characidium rachovii (Regan, 1913) é uma espécie frequente em riachos da planície costeira do estado do Rio Grande do Sul, sul do Brasil. Entretanto, seu nicho trófico é ainda desconhecido. O presente trabalho teve como objetivos: (i) descrever a dieta de $C$. rachovii de três riachos costeiros do RS; (ii) avaliar a influência de um ecótono (mar-riacho) na dieta, comparando dois locais, um próximo do mar e outro distante, e (iii) avaliar o efeito do número de indivíduos analisados na riqueza da dieta. A análise do conteúdo estomacal de 139 indivíduos revelou que a espécie é especializada no consumo de Diptera (estágio aquático) e Amphipoda. Ao mesmo tempo, pode-se considerar que $C$. rachovii apresentou hábito alimentar oportunista, já que, apesar de tal preferência, consumiu grande riqueza de recursos (24 itens), vários deles em baixa frequência e alta abundância. Não houve diferenças na composição da dieta entre os riachos e locais. A riqueza esperada (rarefação) mostrou que o número de indivíduos analisados altera a estimativa de riqueza da dieta em espécies que consomem itens raros.

PALAVRAS-CHAVE. Nicho trófico, alimentação, rarefação, planície costeira, riacho costeiro.

Os riachos de planície costeira do Rio Grande do Sul são sistemas conspícuos ao longo dos cerca de $620 \mathrm{~km}$ de praias arenosas do litoral do estado. Estes riachos também são conhecidos como sangradouros, pois têm importante papel de promover a drenagem das áreas de banhados e das lagoas costeiras na área de pós-dunas, desaguando nas praias oceânicas adjacentes (Figueiredo \& CALLiari, 2005; 2006). Estes ambientes são relativamente bem conhecidos sob os aspectos geomorfológicos (Figueiredo \& CALliari, 2005; 2006). Entretanto, poucos estudos foram realizados com a comunidade biológica dos sangradouros (ALBERTONI \& Palma-Silva, 2006; Seeliger et al., 2004).

O peixe-canivete ou charutinho Characidium rachovii (Regan, 1913) é uma espécie de pequeno porte (comprimento total máximo: $6 \mathrm{~cm}$ ) que habita principalmente pequenos riachos e banhados ao longo das bacias de drenagem do rio Uruguai e da laguna dos Patos, estendendo-se até as zonas baixas da bacia de drenagem do rio da Prata (Uruguai-Argentina), com registro de ponto mais austral próximo a Los Talas, Buenos Aires, Argentina (BUCKUP \& ReIS, 1997). No Rio Grande do Sul esta espécie é frequente nos sangradouros que deságuam na praia do Cassino (BAstos et al., 2013a,b) e foi registrada na laguna dos Patos e área de banhados do Parque Nacional da Lagoa do Peixe (Loebmann \& Vieira, 2005; Corrêa et al.,
2010) e na estação Ecológica do Taim (GArcia et al., 2006).

$\mathrm{Na}$ literatura científica há apenas menções sobre os itens alimentares consumidos por $C$. rachovii (CorrêA et al., 2010) e sobre a frequência de ocorrência destes (Fernandez et al., 2012). Estes estudos preliminares sobre a alimentação de $C$. rachovii (CorrêA et al., 2010; Fernandez et al., 2012) e outros trabalhos com espécies congenéricas (SABINO \& Castro, 1990; Aranha et al., 2000; Motta \& Uieda, 2004; Barreto \& Aranha, 2006; Ferreira, 2007; Gomiero \& Braga, 2008; Braga \& Gomiero, 2009; CETRA et al., 2011; MAZzoni et al., 2012) mostraram uma maior representatividade de insetos imaturos (estágios aquáticos, principalmente larvas de dípteros) e crustáceos na dieta.

Objetiva-se descrever a dieta e estratégia alimentar de Characidium rachovii e avaliar possíveis diferenças dos indivíduos em relação a: 1) diferentes riachos localizados na mesma planície costeira; 2) dentro do mesmo riacho, em um local próximo a sua desembocadura no mar e em outro a montante. Além disso, com o objetivo de verificar a influência do número de indivíduos analisados sobre os resultados, foram comparadas a riqueza observada (S) e a riqueza esperada $(\mathrm{E}[\mathrm{S}])$ das dietas entre os diferentes tratamentos (riachos e locais). 


\section{MATERIAL E MÉTODOS}

O estudo foi realizado na porção sul do litoral do Rio Grande do Sul, Brasil, que possui seu limite norte no molhe oeste da barra do canal de acesso ao porto do município de Rio Grande e estende-se até o arroio Chuí, na fronteira com o Uruguai. As amostragens foram conduzidas em três riachos costeiros (R1, R2 e R3), sendo dois locais amostrados em cada um dos riachos. O primeiro local (ponto $\mathrm{A}$ ) foi definido como a área em que o riacho corta a linha do cordão de dunas frontais, próximo à desembocadura no mar, e o segundo local (ponto B) na região de pós-dunas, cerca de $1 \mathrm{~km}$ riacho acima (Fig. 1). Em cada local foram feitas coletas mensais ao longo de um ano, entre os meses de abril de 2010 e março de 2011.

Em cada ponto de amostragem foram realizados três arrastos de praia paralelos às margens do sangradouro, com rede tipo picaré (nove metros de comprimento, malha multifilamento, $13 \mathrm{~mm}$ nas asas e cinco $\mathrm{mm}$ num pano central sem saco). A área estimada de cada arrasto foi de $40 \mathrm{~m}^{2}$. Também foram realizados três arrastos com uma rede tipo beam trawl, sendo que cada arrasto teve uma área varrida de aproximadamente $8 \mathrm{~m}^{2}$. A rede empregada possui estrutura rígida em formato quadrado $(0,8 \mathrm{~m} \mathrm{x} 0,8$ m) de cano PVC acoplado a um saco fechado em malha multifilamento de $5 \mathrm{~mm}$ entre nós adjacentes. Além das redes de arrasto, foram feitas coletas com puçá $(39 \mathrm{~cm}$ de diâmetro, malha $5 \mathrm{~mm}$ multifilamento), o qual foi operado por 15 minutos em cada local. Devido à dificuldade de padronização de amostragem com puçá entre diferentes pessoas, todas as coletas foram efetuadas pelo mesmo operador. Os indivíduos coletados foram fixados em formol $10 \%$ no campo e, posteriormente, conduzidos para triagem no laboratório, onde os estômagos foram retirados e conservados em álcool $70 \%$ para posterior análise do conteúdo.

Os itens alimentares em cada estômago foram classificados até o menor nível taxonômico possível e contabilizados para o cálculo da Frequência de Ocorrência percentual (FO\%), que expressa o percentual de estômagos em que o item ocorreu (HyNEs, 1950; HYSLOP, 1980). Já o volume ocupado por cada item foi obtido pelo método volumétrico rápido de Hellawell \& Abel (1971), com pequenas modificações. Neste método, os diferentes itens alimentares são separados em grupos (menor nível taxonômico possível) e espalhados sobre uma placa de Petri milimetrada com alturas padronizadas em $1 \mathrm{~mm}$. Para tal, os itens são compactados contra lâminas de vidro de $1 \mathrm{~mm}$ de espessura, pelas laterais e por cima, formando blocos de $1 \mathrm{~mm}^{3}$. Assim, os blocos formados tem seu volume (VOL) mensurado $\left(\mathrm{mm}^{3}\right)$. Posteriormente, foi calculado o Volume percentual (VOL\%), que representa a proporção da soma do volume ocupado pelo item em relação à soma do volume ocupado por todos os itens (considerando todos riachos, riacho por riacho e locais

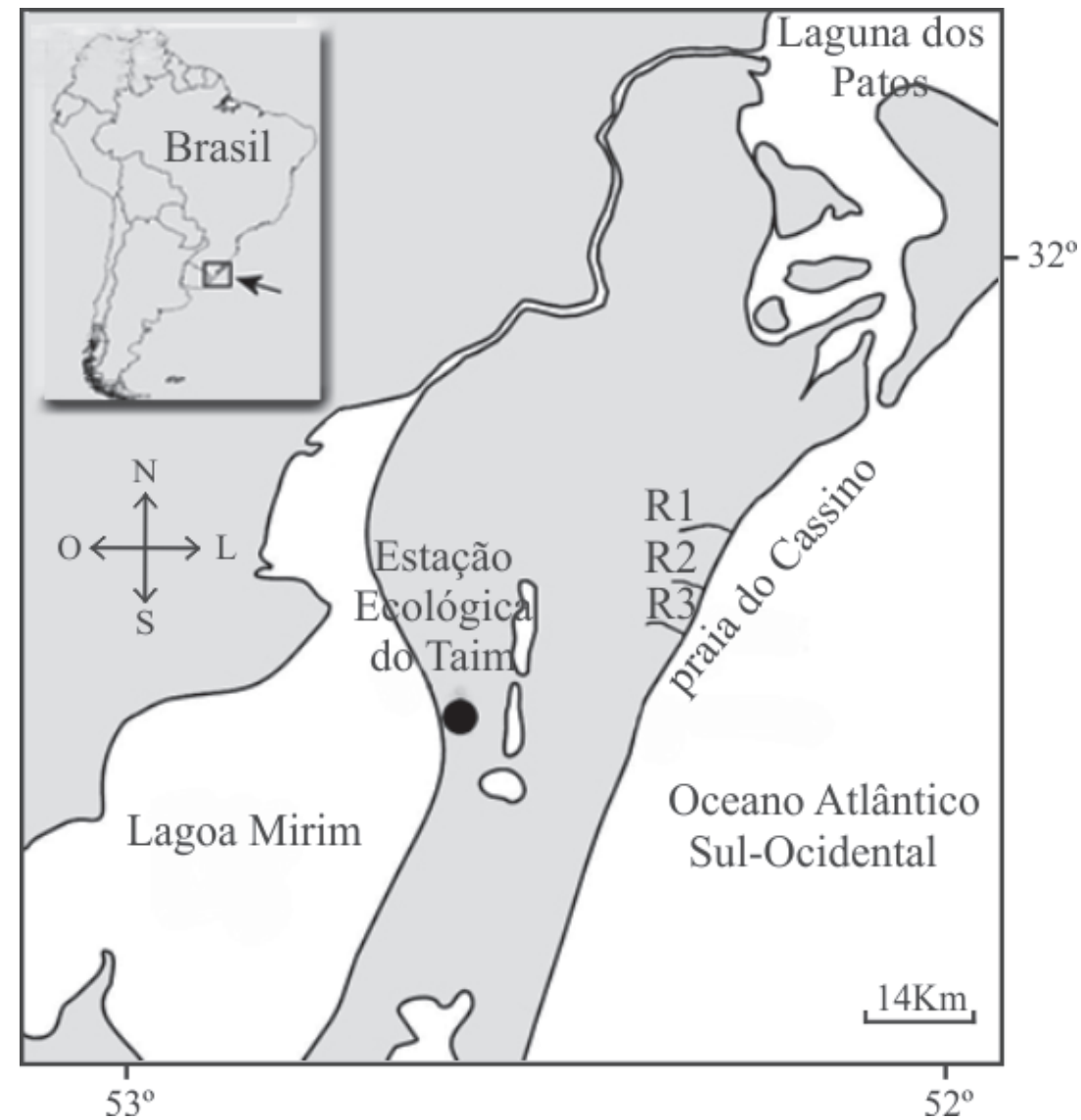

Fig. 1. Mapa da região do litoral do Estado do Rio Grande do Sul (Brasil) com a localização dos três riachos estudados (R1, R2 e R3). 
do riacho 3). A importância de cada item alimentar foi avaliada utilizando o Índice Alimentar (IAi), que é calculado por meio do produto entre $\mathrm{FO} \%$ e VOL\% (KaWAKAMI \& VAZZOLER, 1980).

A estratégia alimentar foi avaliada pelo método gráfico proposto por AMUNDSEN et al. (1996; modificado de Costello, 1990). Essa análise utiliza um plano cartesiano onde são plotados os valores de frequência de ocorrência percentual (FO\%) no eixo das abcissas (x) e a abundância das presas no eixo das ordenadas (y), sendo calculada a abundância de determinada presa considerando apenas os estômagos em que esta ocorreu. Desta maneira, calcula-se a abundância específica percentual (AE\%) pela soma do volume que determinado item ocupou, dividido pela soma do volume ocupado por todos os itens, considerando apenas os estômagos em que o mesmo ocorreu. A disposição dos pontos, que representam cada presa no plano (FO\% x AE\%), permite inferir sobre: (i) importância das presas, no qual as presas dominantes localizam-se no canto superior direito do gráfico, ou seja, com altos valores de $\mathrm{FO} \%$ e $\mathrm{AE} \%$, e as presas raras concentram-se no canto inferior esquerdo, com baixos valores de $\mathrm{FO} \%$ e $\mathrm{AE} \%$; (ii) grau de especialização ou generalização, no qual especialistas possuem suas presas na parte superior do gráfico, com altos valores de $\mathrm{AE} \%$, e generalistas na parte inferior, com baixos valores de $\mathrm{AE} \%$; e (iii) componentes intra e entre fenotípicos da amplitude do nicho, no qual uma população em que diferentes indivíduos se especializam em diferentes tipos de presas possui um alto componente entre fenótipos, e por outro lado, uma população em que a maioria dos indivíduos utilizam diferentes recursos simultaneamente possui um alto componente intra fenótipos. Ambos componentes fenotípicos podem ser visualizados no diagrama, já que presas com abundância específica alta e baixa frequência de ocorrência (localizadas no canto superior esquerdo) foram consumidas por poucos indivíduos que mostram especialização (alto componente entre fenótipos), enquanto presas com alta frequência de ocorrência e baixa abundância específica foram consumidas ocasionalmente, mas pela maioria da população (alto componente intra fenótipos) (GARCIA et al., 2005).

A análise multivariada não-paramétrica de similaridade (ANOSIM) de um fator foi aplicada sobre a matriz dos valores absolutos de volume ocupado pelos itens (VOL) por indivíduo analisado, para testar se a composição da dieta é diferente nos riachos e nos pontos amostrados. O nível de significância foi testado por meio de 10.000 permutações entre os grupos. A estatística " $R$ " do ANOSIM é uma indicação de diferença entre os grupos formados, sugerindo similaridade total entre os grupos $(R=-1)$, formação de grupos ao acaso $(R=0)$ ou dissimilaridade total entre os grupos $(R=1)$. Com o objetivo de complementar a ANOSIM, foi utilizada uma análise de escalonamento multidimensional não métrico (NMDS - Non-metric Multi-Dimensional Scaling). A NMDS apresenta ordenação gráfica num plano cartesiano, em que a distância de cada ponto (amostra) para o outro ponto está inversamente relacionada com a similaridade entre eles, ou seja, quanto maior a distância, menor a similaridade e vice-versa. Para a identificação das presas que mais contribuíram na formação dos grupos, foi utilizada a análise de percentual de similaridade mínima (SIMPER). Para o cálculo de todas as análises multivariadas e de similaridade foi utilizado o pacote PRIMER V. 6 (Clarke \& Gorley, 2006). Para avaliar diferenças na dieta entre os indivíduos capturados nos locais A e B, as amostras dos riachos R1-A $(n=6)$ e R2-A $(\mathrm{n}=1)$ foram excluídas posteriormente, devido ao baixo " $n$ " amostral. Dessa maneira a comparação entre pontos ficou restrita aos indivíduos coletados no riacho R3 (R3$\mathrm{A}, \mathrm{n}=44$; R3-B, $\mathrm{n}=29$ ).

Com o objetivo de avaliar o efeito do tamanho da amostra sobre a riqueza de itens ingeridos pela espécie, foi calculada a riqueza rarefeita, ou riqueza esperada (Gotelli, 2009). Em razão de a abundância numérica dos itens (presas) nos estômagos dos peixes ser de difícil mensuração (muitos itens não são encontrados inteiros ou estão em avançado grau de digestão), considerou-se cada estômago analisado como uma amostra e a quantidade de itens consumidos como o número de indivíduos coletados.

\section{RESULTADOS}

Foram coletados e analisados um total de 139 espécimes de C. rachovii, com medidas entre $30 \mathrm{~mm}$ e $50 \mathrm{~mm}$ de comprimento total. Desses, 21 indivíduos foram coletados no riacho R1, 25 no R2 e 93 no R3. Todos os estômagos continham itens alimentares. No total, 24 itens alimentares foram registrados na dieta (Tab. I). O item alimentar mais importante nos três riachos estudados foi exemplares de Diptera em estágios aquáticos (larvas e pupas), seguido de fragmentos de outros insetos em estágios aquáticos (larvas, pupas e ninfas) e Amphipoda.

A composição da dieta foi semelhante entre os diferentes grupos analisados. Os valores de IAi\% mostraram que os mesmos itens (Diptera, fragmentos de insetos em estágios aquáticos e Amphipoda) aparecem como as principais presas das dietas da espécie quando comparadas entre os riachos (Tab. I). Da mesma maneira, a comparação entre os locais próximos à desembocadura (A) e a montante (B) mostrou que a dieta é muito semelhante ao longo de um mesmo riacho (Tab. I).

$\mathrm{Na}$ comparação multivariada (ANOSIM) da composição da dieta entre os riachos e entre os locais não demonstrou diferenças nem similaridades, com valores de $R$ próximos de zero $\left(R_{\text {riachos }}=-0,053 / p_{\text {riachos }}\right.$ $\left.>0,05 ; \quad R_{\text {locais }}=-0,074 / p_{\text {locais }}<0,05\right)$, o que indica formação de grupos ao acaso. De acordo com a SIMPER, as amostras dos riachos apresentaram baixa similaridade quando comparadas dentro dos mesmos riachos e entre eles. O R1 apresentou similaridade média de $28,21 \%$, no qual Diptera foi o item de maior contribuição $(55,71 \%)$, 
Tab I. Valores do Índice de Importância Alimentar (IAi\%), número amostral, riqueza e riqueza rarefeita dos itens alimentares consumidos por Characidium rachovii (Regan, 1913). Os valores são apresentados considerando as condições: (1) todos os riachos e locais estudados (coluna Geral); (2) cada um dos riachos estudados (colunas R1, R2 e R3); (3) locais individualizados, somente do riacho 3 (R3) (colunas R3-A e R3-B). A riqueza rarefeita por amostra foi calculada pelo menor número de estômagos analisados em cada condição. Os valores de riqueza rarefeita são acompanhados dos respectivos valores de desvio padrão (d.p.). O símbolo “*” representa valor de IAi inferior a 0,1. Espaços em branco representam a ausência do item na condição considerada.

\begin{tabular}{|c|c|c|c|c|c|c|}
\hline Itens alimentares & IAi & & & & & \\
\hline & Geral & R1 & $\mathrm{R} 2$ & $\mathrm{R} 3$ & R3-A & R3-B \\
\hline Diptera (estágios aquáticos) & 48,2 & 55,7 & 42,7 & 45,5 & 45,0 & 44,2 \\
\hline Fragmentos de insetos (estágios aquáticos) & 24,9 & 35,2 & 13,6 & 26,8 & 26,2 & 27,5 \\
\hline Amphipoda & 18,4 & 5,3 & 33,7 & 16,0 & 14,0 & 19,6 \\
\hline Fragmentos de invertebrados indeterminados & 2,8 & & 8,8 & 2,4 & 3,4 & 0,9 \\
\hline Ephemeroptera (estágios aquáticos) & 1,6 & 0,5 & 0,5 & 2,6 & 4,4 & 0,5 \\
\hline Ostracoda & 1,5 & 1,7 & 0,1 & 2,2 & 3,1 & 0,9 \\
\hline Trichoptera (estágios aquáticos) & 0,9 & 0,2 & 0,2 & 1,6 & 1,0 & 2,8 \\
\hline Detrito & 0,7 & 0,4 & & 1,4 & 1,0 & 2,2 \\
\hline Fragmentos de vegetais & 0,4 & & 0,1 & 0,8 & 0,8 & 0,7 \\
\hline Odonata (estágios aquáticos) & 0,2 & 0,2 & 0,1 & 0,1 & 0,4 & \\
\hline Escamas & 0,2 & * & 0,1 & 0,2 & 0,3 & 0,1 \\
\hline Sementes & * & 0,2 & $*$ & 0,1 & 0,2 & $*$ \\
\hline Coleoptera (estágios aquáticos) & $*$ & * & & 0,1 & * & 0,2 \\
\hline Plecoptera (estágios aquáticos) & $*$ & & & 0,1 & 0,2 & \\
\hline Insetos (estágios aquáticos) indeterminados & $*$ & 0,4 & & * & $*$ & \\
\hline Hymenoptera & $*$ & 0,1 & & $*$ & * & 0,1 \\
\hline Copepoda & $*$ & & & $*$ & * & $*$ \\
\hline Hydracarina & $*$ & & $*$ & $*$ & * & \\
\hline Bivalvia & * & 0,1 & & & & \\
\hline Lepidoptera (imaturo) & $*$ & & & $*$ & $*$ & \\
\hline Matéria indeterminada & * & & $*$ & & & \\
\hline Araneae & $*$ & * & & & & \\
\hline Cladocera & $*$ & & & $*$ & * & \\
\hline Alga filamentosa & $*$ & & * & & & \\
\hline Número de estômagos analisados & 139 & 21 & 25 & 93 & 61 & 32 \\
\hline Riqueza & 24 & 15 & 14 & 20 & 20 & 14 \\
\hline Riqueza rarefeita por amostra (+-d.p.) & - & $\begin{array}{c}15 \\
(+-2,7)\end{array}$ & $\begin{array}{c}13,4 \\
(+-1,0)\end{array}$ & $\begin{array}{c}13,9 \\
(+-1,8)\end{array}$ & $\begin{array}{c}16,8 \\
(+-1,8)\end{array}$ & $\begin{array}{c}14 \\
(+-1,4)\end{array}$ \\
\hline
\end{tabular}

seguido por fragmentos de outros insetos $(33,24 \%)$ e Amphipoda (6,93\%). O R2 apresentou similaridade média de 22,62\%, com Diptera (36\%), Amphipoda $(31,99 \%)$, fragmentos de outros insetos $(16,05 \%)$ e fragmentos de invertebrados não identificados $(12,85 \%)$ os responsáveis por $90,01 \%$ dessa similaridade. O R3 foi o riacho com menor similaridade $(17,61 \%)$, sendo que os itens, Diptera $(36,84 \%)$, fragmentos de outros insetos $(28,96 \%)$, Amphipoda $(20,15 \%)$ e Ostracoda $(4,07 \%)$ foram os responsáveis por $90,01 \%$ dessa similaridade. Quando as amostras foram comparadas entre os riachos, foram verificadas dissimilaridades entre $77,8 \%$ (R1 e R3) e 81,28 (R2 e R3), passando por 78,3 (R1 e R2). Todas as dissimilaridades tiveram como princiais itens Diptera (R1xR2: 24,7\%; R1xR3: $23,06 \%$ e R2xR3: 21,38\%), fragmentos de outros insetos (R1xR2: 19,53\%; R1xR3: $23,73 \%$ e R2xR3: 19,06\%) e Amphipoda (R1xR2: 17,31\%; R1xR3: 13\% e R2xR3: 17,78\%).
A análise da estratégia alimentar revelou não ter grandes diferenças entre os riachos (Figs 2-4). Da mesma maneira, os pontos comparados dentro do riacho R3 foram muito similares nos recursos consumidos pela maioria dos espécimes (Figs 6, 7), em que apenas se destacou a maior abundância específica de alguns ites no ponto A (Fig. 6). De modo geral, considerando todos os espécimes analisados independentemente do riacho ou ponto, a análise da estratégia alimentar mostra os itens mais importantes no centro do gráfico, o que indica uma preferência por cerca de metade da população em insetos (formas aquáticas imaturas, principalmente de dípteros) e anfípodos (Fig. 5). Apesar disso, muitos itens se concentram na porção inferior esquerda, indicando um consumo casual de presas raras e poucos itens na porção superior esquerda, indicando consumo oportunista destas presas por poucos indivíduos (Fig. 5). Na porção superior direita nenhuma presa é encontrada, 
o que significa que a população estudada não é altamente especializada em qualquer recurso específico.

A riqueza observada $(\mathrm{S})$ na dieta dos indivíduos foi influenciada pelo número de estômagos analisados (Tab. I). O riacho $\mathrm{R} 3$, que teve o maior número de estômagos analisados (93), foi aquele com a maior riqueza observada $(\mathrm{S}=20)$ quando comparado aos demais riachos $(\mathrm{R} 1: \mathrm{S}=15 ; \mathrm{N}=21$ e $\mathrm{R} 2: \mathrm{S}=14$; $\mathrm{N}$ $=25$ ). Entretanto, quando as diferenças no número de amostras (número de estômagos analisados) entre os riachos foi padronizado pelo método de rarefação $(\mathrm{N}$ = 21 amostras), não foi possível observar diferenças na riqueza esperada $(\mathrm{E}[\mathrm{S}])$ da dieta da espécie entre os riachos (Tab. I). Um padrão similar foi obsevado em relação aos dois locais do riacho R3. O local A teve um maior número de indivíduos analisados $(\mathrm{N}=61)$ e apresentou riqueza observada $(\mathrm{S})$ maior $(\mathrm{S}=20)$ quando comparado ao local $\mathrm{B}(\mathrm{N}=32 ; \mathrm{S}=14)$. Porém, quando as diferenças no número de amostras foram padronizadas pelo menor número de estômagos analisados $(\mathrm{N}=32)$, as diferenças na riqueza entre os dois locais desaparecem (Tab. I).

\section{DISCUSSÃO}

Characidium rachovii apresentou hábito alimentar invertívoro com forte tendência a larvivoria, sendo que suas principais presas consistiram em formas aquáticas imaturas de insetos - principalmente dípteros -, e de anfípodos. Outras espécies congenéricas mostraram padrão semelhante, em que larvas e formas imaturas aquáticas de insetos compuseram a maior parte da dieta; microcrustáceos e outros invertebrados ocorreram mais raramente (SABINO \& CASTRO, 1990; CASTRO \& CASATTI, 1997; UiEDA et al., 1997; ARANHA et al., 2000; BARRETO \& Aranha, 2006; Ferreira, 2007; Gomiero \& BraGA, 2008). Quando avaliamos a estratégia alimentar e a contribuição dos indivíduos para a amplitude do nicho trófico da espécie, é possível observar que metade da população (entre 40 e 60\% dos indivíduos analisados) consumiu principalmente formas imaturas de dípteros e anfípodos em todos os riachos estudados, com valores de abundância específica da presa (AEP) em torno de 20 a $50 \%$.

Entretanto, a elevada riqueza de itens na dieta,

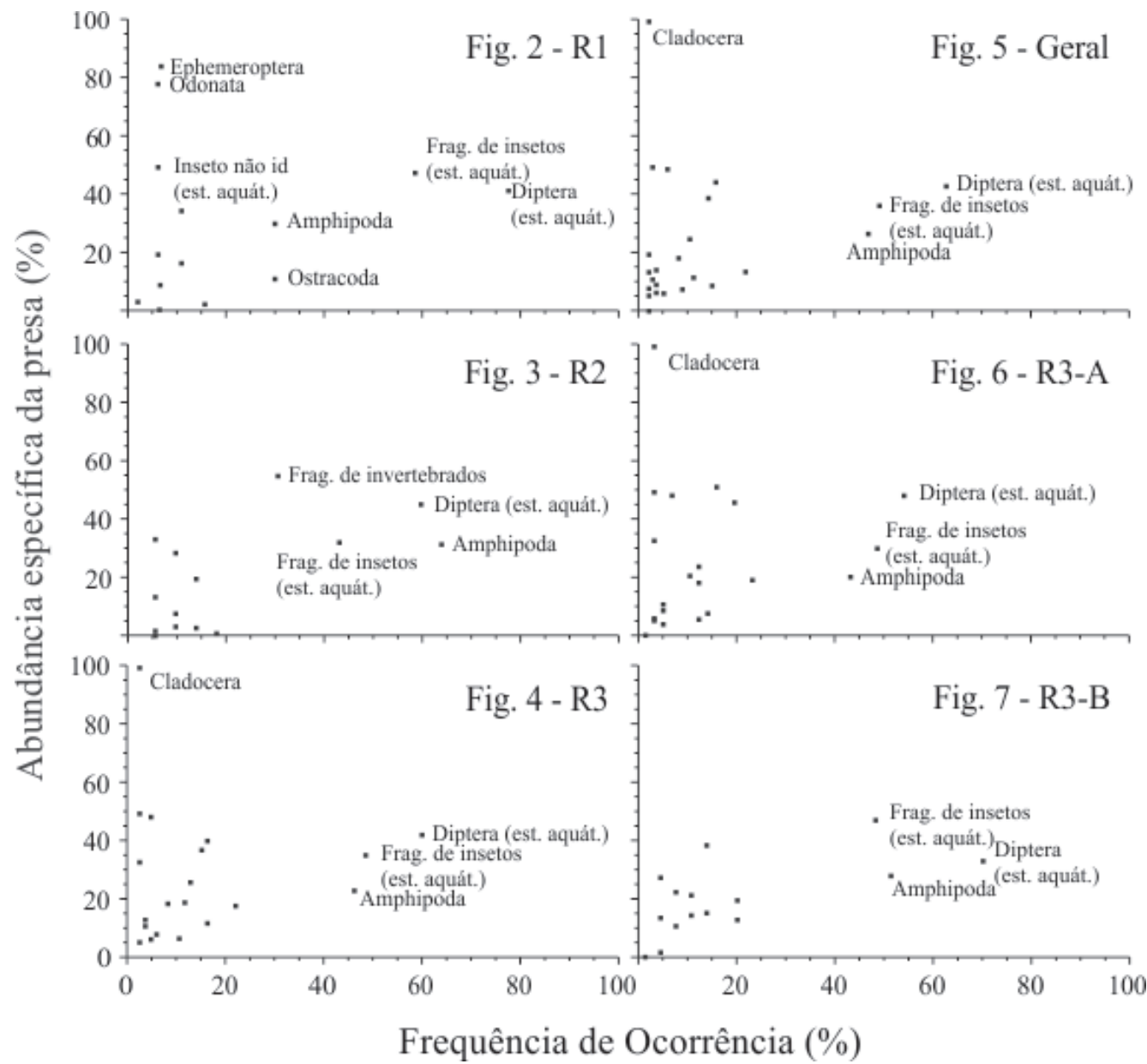

Figs 2-7. Valores de Frequência de Ocorrência percentual - FO\% (eixo x) e Abundância Específica percentual - AE\% (eixo y) dos itens alimentares encontrados nos estômagos de Characidium rachovii (Regan, 1913) em cada condição analisada. Na condição de comparação entre riachos, $\mathrm{R} 1, \mathrm{R} 2$ e R3 representam os valores $(\mathrm{FO} \%$ e $\mathrm{AE} \%)$ dos itens para cada riacho. O gráfico geral ilustra os valores dos itens alimentares consumidos pela espécie agrupando todos os riachos. R3-A e R3-B representam os valores encontrados para os indivíduos capturados em cada um dos locais (A e B) no riacho 3 (est. aquát., estágios aquáticos). 
associada à baixa frequência de ocorrência (FO\%) e baixa abundância especifica ( $\mathrm{AE} \%$ ) da maioria das presas mostra que os indivíduos da população de C. rachovii dos riachos estudados consomem muitos itens raros. Apesar disso, três itens alimentares ocorreram na dieta de cerca de metade dos indivíduos analisados e ocuparam aproximadamente a metade do volume dos estômagos em que ocorreram, indicando uma estratégia alimentar do tipo especialista, pois, apesar de consumir grande riqueza de itens, indivíduos mostraram preferência sobre formas aquáticas imaturas de insetos e anfípodos.

Além disso, a maioria dos itens raros (baixa $\mathrm{FO} \%$ ) foi consumida em baixa abundância específica (AE\%) e poucos tiveram alta $\mathrm{AE} \%$, o que indica um grande consumo de itens raros na população e o oportunismo e/ ou especialização de poucos indivíduos. O oportunismo alimentar e a ocorrência de diversos itens raros na dieta já foi descrito anteriormente para diferentes espécies de Characidium (Aranha et al., 2000). Portanto, além do caráter especialista, a espécie se mostrou oportunista, predando sobre muitos recursos alimentares, mas mais intensamente sobre poucos grupos.

É interessante observar, entretanto, que pequena parcela da população $(<10 \%$ dos indivíduos) consumiu elevadas quantidades (AEP $\geq 80 \%$ ) de efemerópteros e odonatos no riacho $\mathrm{R} 1$ e cladóceros no riacho $\mathrm{R} 3$ (ponto A). Desse modo, embora a população de Characidium rachovii analisada possua uma dieta com predomínio de insetos imaturos e anfípodos, é possível perceber certa contribuição do componente interfenotípico (sensu AMUNDSEN et al., 1996) no nicho trófico da espécie. Ou seja, o nicho trófico de C. rachovii nos riachos litorâneos do sul do Rio Grande do Sul é composto na sua maior parte por indivíduos que consomem insetos imaturos e anfípodos, mas também por uma pequena parcela de indivíduos na população que, de maneira oportunista ou por especialização individual, consomem efemerópteros, odonatos e cladóceros.

Esses resultados reforçam a teoria de que mesmo em espécies com hábito alimentar aparentemente restrito ao consumo de um ou dois itens particulares, como no presente estudo, é possível observar variações individuais no comportamento alimentar. Por exemplo, BolNiCK et al. (2003) argumentam que a incidência da especialização individual no consumo de presas tem sido historicamente negligenciada em estudos de dieta e ecologia trófica de predadores como os peixes.

Segundo MotTA \& UiEDA (2004), a predominância de insetos aquáticos na dieta de Characidium schubarti Travassos, 1955 pode estar relacionada com a utilização de macrófitas aquáticas como local de alimentação, pois este microhabitat é colonizado por uma entomofauna rica e abundante, que o utiliza como local de abrigo e alimentação. BARRETO \& ARANHA (2006) identificaram, para Characidium lanei Travassos, 1967, um comportamento de localização, espreita e investida sobre o alimento, principalmente larvas, ou de procura junto ao fundo de areia, caracterizando os indivíduos estudados como catadores. Albertoni \& PAlmaSilva (2006) encontraram dípteros (forma aquática imatura) como o macroinvertebrado mais abundante em macrófitas flutuantes em riachos próximos aos da área do presente estudo e com características similares. Assim, a prevalência de dípteros na dieta de $C$. rachovii nos riachos pode estar relacionada à abundância deste no ambiente e, principalmente, ao microhabitat explorado pela espécie (macrófitas e fundo arenoso).

As análises dos valores de IAi\% e dos valores de R (ANOSIM) mostraram não haver diferenças ou semelhanças marcantes na composição da dieta entre os riachos e locais estudados, indicando uma formação de grupos ao acaso. Essa padrão reitera o caráter oportunista da espécie que frequentemente consome itens raros.

A técnica de rarefação foi desenvolvida no final da década de 1960 (SANDERS, 1968) e tem sido aplicada extensivamente em estudos sobre a diversidade de assembleias e/ou comunidades (GARCIA \& VIEIRA, 1997; ESPIRITO-SANTO et al., 2009) e, em menor extensão, em estudos sobre dieta de animais (KovÁCs \& TöRÖK, 1997; CAstillo et al., 2007; Bortolini et al., 2013). Essa técnica torna-se importante quando estamos comparando a riqueza de itens na dieta de uma espécie entre situações, tais como locais e períodos, com um número desigual de estômagos analisados (KovÁcs \& TöRÖK, 1997). O presente estudo revelou que o número de amostras pode influenciar a riqueza observada (S) na dieta de C. rachovii em riachos da planície costeira do RS. Assim, espécies que consomem itens de maneira oportunista ou que apresentam preferências indivíduais, como neste caso, vão apresentar uma riqueza na dieta observada diretamente proporcional ao número de estômagos com conteúdo analisados. Esse resultado reforça a importância da padronização do esforço amostral, ou a utilização de técnicas que diminuam o efeito do número desigual de amostras, tal como a técnica de rarefação, para evitar interpretações e conclusões errôneas em estudos de riqueza na dieta.

De maneira geral, C. rachovii apresentou um nicho alimentar semelhante ao descrito para as espécies congenéricas. O hábito de forragear sobre a comunidade bentônica/fitobentônica de riachos e lagoas parece ser um padrão de Characidium. A preferência sobre formas imaturas de insetos imaturas e microcrustáceos, tais como os anfípodos, aqui registrada, provavelmente está ligada à maior disponibilidade desses recursos nos locais estudados aliada à acessibilidade desses para a espécie. Além disso, o oportunismo alimentar ficou evidente pelo consumo de uma ampla gama de recursos, ainda que em pouca quantidade. Por fim, este estudo mostrou que a riqueza da dieta de C. rachovii está diretamente relacionada com o número de amostras (estômagos analisados com conteúdo) e, portanto, para comparações entre agrupamentos com diferentes número de amostras, recomenda-se a utilização da riqueza rarefeita ou outros outros procedimentos que padronizem o esforço amostral entre os grupos. 
AGRADECIMENTOS. Os autores agradecem à Comissão de Aperfeiçoamento de Pessoal de Nível Superior (CAPES), ao programa de Reestruturação das Universidades Federais (REUNI) e ao Conselho Nacional de Pesquisa (CNPq), pelas bolsas concedidas; aos colegas do laboratório de Ictiologia (IO - FURG), pelo auxílio nas coletas e no processamento das amostras e a Leonardo Moraes, Júlio César Bicca-Marques e um aluno anônimo do Programa de Pós Graduação em Zoologia - PUCRS, pelas sugestões e críticas.

\section{REFERÊNCIAS BIBLIOGRÁFICAS}

Albertoni, E. F. \& Palma-Silva, C. 2006. Macroinvertebrados associados a macrófitas aquáticas flutuantes em canais urbanos de escoamento pluvial (Balneário Cassino, Rio Grande, RS). Neotropical Biology and Conservation 1(2):90-100.

Amundsen, P. A.; Gabler, H. M. \& Staldvik, F. J. 1996. A new approach to graphical analysis of feeding strategy from stomach contents data - Modification of the Costello (1990) method. Journal of Fish Biology 48:607-614.

Aranha, J. M. R.; Gomes, J. H. C. \& FogaçA, F. N. O. 2000. Feeding of two sympatric species of Characidium, C. lanei and C. pterostictum (Characidiinae) in a coastal stream of Atlantic Forest (Southern Brazil). Brazilian Archives of Biology and Technology 43(5):527-531.

Barreto, A. P. \& Aranha, J. M. R. 2006. Diet of four species of Characiforms in an Atlantic forest stream, Guaraquecaba, Paraná, Brazil. Revista Brasileira de Zoologia 23(3):779-788

Bastos, R. F.; Calliari, L. J. \& Garcia, A. M. 2013a. Marine intrusion and freshwater discharge as opposite forces driving fish guilds distribution along coastal plain streams. Hydrobiologia. online first. doi:10.1007/s10750-013-1771-7. Avaliable at: $<$ http:/ download.springer.com/static/pdf/208/art\%253A10.1007\%252 Fs10750-013-1771-7.pdf?auth66=1389790920 a8cd0233ac85b6 6e93fdd96159c46dda\&ext=.pdf $>$. Accessed on: 10.01.2014.

Bastos, R. F.; Condini, M. V. \& Garcia, A. M. 2013b. Fish species list of coastal streams in southern Brazil, with notes on austral distribution limits of marine and freshwater endangered species. Pan-American Journal of Aquatic Sciences 8(4):347-351

Bolnick, D. I.; SvanbäcK, R.; Fordyce, J. A.; Yang, L. H.; Davis, J. M.; Hulsey, C. D. \& Forister, M. L. 2003. The ecology of individuals: incidence and implications of individual specialization. The American Naturalist 161(1):1-28.

Bortolini, S. V.; Maneyro, R.; Coppes, F. A. \& Zanella, N. 2013. Diet of Melanophryniscus devincenzii (Anura: Bufonidae) from Parque Municipal de Sertão, Rio Grande do Sul, Brazil. The Herpetological Journal 23(2):115-119.

Braga, F. M. DE S. \& Gomiero, L. M. 2009. Alimentação de peixes na microbacia do Ribeirão Grande, Serra da Mantiqueira oriental, SP. Biota Neotropica 9(3):2017-2012.

Buckup, P. A. \& ReIS, R. E. 1997. Characidiin Genus Characidium (Teleostei, Characiformes) in Southern Brazil, with Description of Three New Species. Copeia 1997(3):531-548.

Castillo, K.; Ibanez, C. M.; Gonzalez, C. \& Chong, J. 2007. Diet of swordfish Xiphias gladius Linnaeus, 1758 from different fishing zones off central-Chile during autumn 2004. Revista de Biologia Marina y Oceanografia 42(2):149-156.

Castro, R. M. C. \& CASatTi, L. 1997. The fish fauna from a small forest stream of the upper Paraná River basin, southeastern Brazil. Ichthyological Exploration of Freshwaters 7(3):337-352.

Cetra, M.; Rondineli, G. R. \& Souza, U. P. 2011. Compartilhamento de recursos por duas espécies de peixes nectobentônicas de riachos na bacia do rio Cachoeira (BA). Biota Neotropica 11(2):87-95.

Clarke, K. R. \& Gorley, R. N. 2006. Primer v. 6: User manual/ tutorial. Plymouth, PRIMER-E Ltd. 188p.

CorrêA, F.; Claudino, M. C. \& Garcia, A. M. 2010. Guia fotográfico e aspectos da biologia dos principais peixes de água doce do Parque Nacional da Lagoa do Peixe, RS. Cadernos de Ecologia Aquática 5(1):28-43.
Costello, M. J. 1990. Predator feeding strategy and prey importance: a new graphical analysis. Journal of Fish Biology 36:261-263.

Espirito-Santo, H. M. V.; Magnusson, W. E.; Zuanon, J.; Mendonça, F. P. \& LANDEIRO, V. L. 2009. Seasonal variation in the composition of $f$ ish assemblages in small Amazonian forest streams: evidence for predictable changes. Freshwater Biology 54(3):536-548.

Fernandez, E. M.; Ferriz, R. A.; Bentos, C. A. \& Lopez, G. R. 2012. Dieta y ecomorfología de la ictiofauna del arroyo Manantiales, provincia de Buenos Aires, Argentina. Revista del Museo Argentino de Ciencias Naturales 14(1):1-13.

FERREIRA, K. M. 2007. Biology and ecomorphology of stream fishes from the rio Mogi-Guaçu basin, Southeastern Brazil. Neotropical Ichthyology 5(3):311-326.

Figueiredo, S. A. \& CAlliari, L. J. 2005. Sangradouros: distribuição espacial, variação sazonal, padrões morfológicos e implicações no gerenciamento costeiro. Gravel 3:45-57.

2006. Washouts in the central and northern littoral of Rio Grande do Sul state, Brazil: Distribution and implications. Journal of Coastal Research SI39:366-370.

Garcia, A. M.; Bemvenuti, M. A.; Vieira, J. P.; Marques, D. M. L. M.; Burns, M. D. M.; Moresco, A. \& Condini, M. V. L. 2006. Checklist comparison and dominance patterns of the fish fauna a Taim Wetland, South Brazil. Neotropical Ichthyology 4(2):261-268.

Garcia, A. M.; Geraldi, R. M. \& VieIRA, J. P. 2005. Diet composition and feeding strategy of the southern pipefish Syngnathus follett in a Widgeon grass bed of the Patos Lagoon Estuary, RS, Brazil. Neotropical Ichthyology 3(3):427-432.

Garcia, A. M. \& VieIRA, J. P. 1997. Abundance and diversity of fish assemblages inside and outside a bed of Ruppia maritima L., in the Patos Lagoon estuary (RS-Brazil). Atlântica 19:161-181.

Gomiero, L. M. \& Braga, F. M. S. 2008. Feeding habits of the ichthyofauna in a protected area in the state of São Paulo, southeastern Brazil. Biota Neotropica 8(1).

Gotelli, N. J. 2009. Ecologia. 4 ed. Londrina, Editora Planta. 288p.

Hellawell, J. M. \& ABEL, R. 1971. A rapid volumetric method for the analysis of the food of fishes. Journal of Fish Biology 3(1):29-37.

HyNeS, H. B. N. 1950. The food of freshwater sticklebacks (Gasterosteus aculeatus and Pygosteus pungitius) with a review of methods used in studies of the food of fishes. Journal of Animal Ecology 19(1):36-58.

Hyslop, E. J. 1980. Stomach contents analisys; a review of methods and their application. Journal of Fish Biology 17(4):411-429.

KaWAKami, E. \& Vazzoler, G. 1980. Método gráfico e estimativa de índice alimentar aplicado no estudo de alimentação de peixes. Boletim do Instituto Oceanográfico de São Paulo 29:205-207.

Kovács, T. \& TöRöK, J. 1997. Determination of minimum sample size to estimate diet diversity in anuran species. The Herpetological Journal 7(2):43-47.

Loebmann, D. \& VieIRA, J. P. 2005. Distribuição espacial e abundância das assembléias de peixes no Parque Nacional da Lagoa do Peixe, Rio Grande do Sul, Brasil. Zoologia 22(3): 667-675.

Mazzoni, R.; Marques, P. S.; Rezende, C. F. \& Iglesias-Rios, R. 2012. Niche enlargement as a consequence of co-existence: a case study. Brazilian Journal of Biology 72(2):267-274.

Motta, R. L. \& Uieda, V. S. 2004. Dieta de duas espécies de peixes do Ribeirão do Atalho, Itatinga, SP. Revista Brasileira de Zoociências 6(2):191-205.

SAbino, J. \& CASTro, R. M. C. 1990. Alimentação, período de atividade e distribuição espacial dos peixes de um riacho da Floresta Atlântica (sudeste do Brasil). Revista Brasileira de Biologia 50(1):23-36.

SANDERs, H. 1968. Marine Benthic Diversity: A Comparative Study. The American Naturalist 102(925):243-283.

Seeliger, U.; Cordazzo, C. \& Barcellos, L. 2004. Areias do Albardão: Um guia ecológico do litoral no extremo sul do Brasil. Rio Grande, Ecoscientia. 96p.

Uieda, V. S.; Buzzato, P. \& KikUChi, R. M. 1997. Partilha de recursos alimentares em peixes em um riacho de serra do Sudeste do Brasil. Anais da Academia Brasileira de Ciências 69:243-252. 